http://dx.doi.org/10.35381/e.k.v4i1.1460

\title{
Herramientas digitales lúdicas para la enseñanza de Lengua y Literatura en
} Escuelas Pluridocentes

Playful digital tools for the teaching of Language and Literature in Pluridocent Schools

\author{
Verónica Mercedes Sarmiento-Robles \\ veronica.sarmiento.66@est.ucacue.edu.ec \\ Universidad Católica de Cuenca, Cuenca \\ Ecuador \\ https://orcid.org/0000-0003-4613-7064 \\ Luis Bolívar Cabrera-Berrezueta \\ bolivarcabrera@ucacue.edu.ec \\ Universidad Católica de Cuenca, Cuenca \\ Ecuador \\ https://orcid.org/0000-0002-6853-635X
}

Recepción: 10 de agosto 2021 Revisado: 15 de septiembre 2021 Aprobación: 15 de noviembre 2021 Publicación: 01 de diciembre 2021 


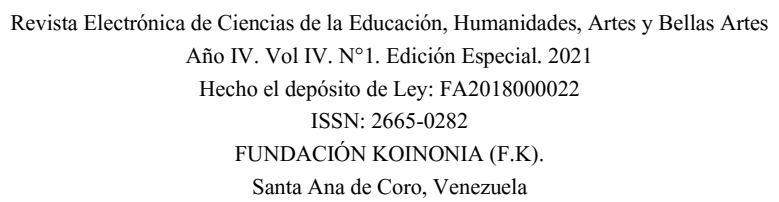

Verónica Mercedes Sarmiento-Robles; Luis Bolívar Cabrera-Berrezueta

\title{
RESUMEN
}

El empleo de estrategias recreativas combinadas con entornos virtuales para que los docentes lo utilicen en las instituciones y pasen de una educación tradicional a una educación moderna. El objetivo general de este proyecto fue analizar de qué manera el uso de herramientas digitales lúdicas pueden aportar a la enseñanza de Lengua y Literatura en Escuelas Pluridocentes, siendo un trabajo de tipo estadístico descriptivo, exploratorio, de campo, bibliográfico y no experimental. Los resultados de la investigación del trabajo demostraron que las herramientas digitales lúdicas ayudan en gran parte para la enseñanza, pero que faltan capacitaciones prácticas, por ello se realizó una propuesta que se basó en una capacitación práctica a docentes sobre herramientas virtuales lúdicas, finalmente se concluye que las herramientas virtuales en base a juegos educativos son una de las mejores opciones para una enseñanza de calidad, en donde se puede incluir contenidos y temas de gran interés.

Descriptores: Juego educativo; material didáctico; material de lectura. (Palabras tomadas del Tesauro UNESCO).

\begin{abstract}
The use of recreational strategies combined with virtual environments for teachers to use in institutions and move from a traditional education to a modern education. The general objective of this project was to analyze how the use of recreational digital tools can contribute to the teaching of Language and Literature in Pluridocent Schools, being a descriptive, exploratory, field, bibliographic and non-experimental statistical type of work. The results of the research of the work showed that playful digital tools help to a large extent for teaching, but that practical training is lacking, therefore a proposal was made that was based on a practical training for teachers on recreational virtual tools, it is finally concluded that virtual tools based on educational games are one of the best options for quality teaching, where content and topics of great interest can be included.
\end{abstract}

Descriptors: Educational games; teaching materials; reading materials. (Words taken from the UNESCO Thesaurus). 


\section{INTRODUCCIÓN}

En la actualidad la enseñanza a través de medios digitales se ha convertido en una de las opciones más favorables que poseen las instituciones educativas para predisponer una adecuada enseñanza, debido a que el contexto ecuatoriano, enfrenta una crisis sanitaria por la pandemia COVID-19 en todos los niveles, pues no sólo es la educación la afectada, también es el ámbito económico, laboral, empresarial y hoy en día con mayor fuerza el de la salud. Dicha realidad ocasiona reacciones diferentes en la población que de forma directa o indirecta repercute en los docentes y estudiantes interfiriendo en su proceso formativo educativo.

Es relevante mencionar que esta investigación busca implantar conocimientos válidos ya que la parte lúdica no es simplemente una actividad espontánea o ligera sino que además envuelve al alumno para que cumpla cada uno de sus objetivos planteados y posteriormente desarrolle de manera óptima sus potencialidades en conjunto a la socialización con otros niños en el entorno escolar; de tal modo que al implementarse como una adaptación curricular favorecería el aprendizaje significativo, también estimula su imaginación y pensamiento lógico.

Por su parte, (CEPAL \& UNESCO, 2020), indican entre los cambios en el currículo que se suscitaron, también se consideraron actividades coherentes considerando las limitaciones físicas de los educandos y el acceso a elementos, por lo que se han incluido usos de plataformas o aplicaciones que les permitan a los educandos interacción con la tecnología, pero también con el conocimiento, entre ellas se han incorporado los juegos en línea, fundamentalmente aquellos que le permiten al niño un aprendizaje significativo, en base a metodologías recreativas, pero el conocimiento del docente respecto a estas herramientas aún sigue siendo limitado, debido a la falta de capacitación.

De esta forma el (Ministerio de Educación, 2020), afirmó también que es necesaria la incorporación de nuevas propuestas pedagógicas para así poder continuar garantizando una formación de calidad, también expuso la importancia de dotar a los educandos de 
herramientas digitales para facilitar su proceso de aprendizaje lo que incluye plataformas, aplicaciones, páginas, blogs, entre otros, debido a las grandes falencias que se han venido presentando a lo largo de estos últimos años.

En la ciudad de Cuenca se han realizado algunos estudios en relación a esta temática, ya que se ha ido considerando mucho más la importancia del uso de la tecnología en la Educación Básica, Media o Superior; puesto que desde el Ministerio de Educación de nuestro país han puesto énfasis en su desarrollado y empleo de entornos digitales rompiendo barreras de los roles comunes alrededor de un pizarrón y un discurso. En este contexto se considera que todavía el docente no logra implementar el manejo adecuado de medios digitales de manera lúdica en el proceso de enseñanza y aprendizaje (Cabrera-Baculima, 2016).

El interés hacia realizar este articulo surge desde el acercamiento previo a la realidad educativa debido a situaciones que obstaculizan los aprendizajes en los estudiantes como es la falta de manejo de herramientas tecnológicas innovadoras, mal uso de estrategias metodológicas lúdicas y falta de capacitación en plataformas virtuales, mismas que se presentan en las Escuelas Pluridocentes de las Parroquias rurales.

En un ambiente digital la enseñanza lúdica debería ser el método más utilizado tanto dentro de una Institución Educativa como fuera de ella, puesto que tiene la capacidad de captar la atención de los estudiantes, organizar en varias formas con el uso de algunas estrategias o herramientas obteniendo resultados positivos al momento de su evaluación, captando la atención de los alumnos por lo que resulta vital la articulación entre todas las actividades pedagógicas con enfoques digitales.

Ante tal situación se torna urgente el accionar estratégico de las instancias educativas, incorporando en sus planificaciones a todos los actores de la educación digital, pero profundizando en el educando pues es éste el que no debe perder el hilo conector con el aprendizaje, por lo que se precisa que su relacionamiento con ellas sea el adecuado, se mantenga la motivación y finalmente se logren aprendizajes significativos. 
Es por ello que se ha considerado pertinente realizar la siguiente investigación que se desarrolla en Ecuador, en la Provincia del Azuay en la Parroquia de Checa, Circuito 1819, en las Escuelas Pluridocentes, con el objetivo de analizar de qué manera el uso de herramientas Digitales Lúdicas aportan a la enseñanza de Lengua y Literatura, con ello se busca dar a conocer a la comunidad escolar cada una de las herramientas y prácticas efectivas que servirán al estudiante al momento de enfrentarse a situaciones que involucra el proceso de enseñanza-aprendizaje dentro del contexto actual.

\section{Referencial teórico}

Anteriormente los docentes se formaban limitaciones únicamente en trasmitir los contenidos establecidos, pero no se tomaba en cuenta el lograr un desarrollo mediante actividades motivadoras, que aporten al crecimiento del pensamiento científico en los estudiantes para que a su vez se tome en consideración una educación activa y netamente participativa; de este modo se ha propiciado el aprendizaje con el empleo de estrategias metodológicas actuales y tecnológicas.

En este sentido, (Adams et al. 2017), en Texas, con referencia a la educación plantea que puede ser innovadora si se implementa herramientas tecnológicas, que ha futuro será un gran desafío tanto para docentes, como estudiantes y que tendrá una gran aprobación positiva en entornos educativos presenciales, ya que antes solo tenía importancia en estudios a distancia, por ello propone dos herramientas que serían las más adecuadas y adaptadas en un periodo a corto plazo, las mismas que pueden ser un aprendizaje adaptativo y un aprendizaje móvil.

Para (Raso-Sánchez et al. 2014), en España, Descubrieron que los docentes tenían algunas habilidades tecnológicas, pero no ponían en práctica en el aula, se guiaban por usar métodos tradicionalistas, dejando a lado habilidades y destrezas en entornos virtuales, por ello el índice de rendimiento de los estudiantes en aspectos educativos y 
didácticos era bajo. Indicaron también que para un buen manejo de competencias digitales los educadores necesitan mayor formación y capacitación.

De igual forma, (Villafuerte, 2020), en sus aportes realizados en México, indica que el docente es el conocedor de sus estrategias metodológicas, materiales, herramientas tecnológicas empleadas con los estudiantes ya sea si la educación es a distancia o presencial deben estar familiarizados tanto docentes como alumnos con el uso de la tecnología.

El uso de herramientas tecnológicas ha empujado a toda la sociedad a un gran cambio donde la educación sucede en un entorno netamente digital debido a la pandemia por COVID-19 que desde hace un año modificó las prácticas educativas. Cada postulado permite al educador basar sus acciones pedagógicas en fundamentos claros y de gran valor en el desarrollo del individuo de manera que se puede reescribir el modelo de educación en la actualidad por medio de la incorporación de las TIC, potenciando la experiencia del educando en los entornos virtuales para un aprendizaje significativo que le permita una verdadera interiorización de la información y desarrollar nuevos conocimientos.

De la misma forma otra investigación planteada por (Camargo-Miranda et al. 2017), en Colombia, para conocer si la implementación de estrategias lúdicas motivaría a los estudiantes a continuar en la jornada diaria y obtener un aprendizaje de calidad, en lo cual se tuvo como resultado la demostración que el fracaso escolar y hasta la deserción estudiantil era causada por la poca implementación de estrategias motivadoras en entornos digitales o a distancia, complementado con la carencia de apoyo y varios factores socioeconómicos.

En relación a la problemática planteada han existido diversas investigaciones desarrolladas acerca de las estrategias metodológicas con actividades lúdicas, (Albornoz \& Guzman, 2016), en Ecuador, menciona que mediante la participación de una estrategia didáctica basada en el juego que sea implementada en el aula, cada uno de los niños 
lograron obtener varios avances significativos en el desarrollo de habilidades tales como: el habla y la afectividad puesto a que reconocieron que el lenguaje oral sirve plenamente como un medio de expresión que ayuda al individuo poder enunciar ideas, sentimientos y participar de forma activa en ciertas conversaciones hasta poder dialogar de manera espontánea.

Por su parte, (Piedra-Vera, 2018) en Ecuador, realizó una investigación que destaca como evidencia a más de 30 factores que colaboran de manera positiva las actividades lúdicas dentro de los contextos educativos, caracterizándose de igual forma por resaltar la creatividad y el aprendizaje significativo correspondiendo a las competencias técnicas dentro del ámbito digital; es por ello que la participación y la socialización han estado presentes de manera continua o frecuente en lo que respecta a las competencias académicas, es decir aprender a conocer y hacer de manera digital.

Respecto a los autores antes mencionados sostienen en sus investigaciones, que la manera de llegar a un aprendizaje de calidad en los niños la base fundamental es mediante el uso de estrategias metodológicas lúdicas, combinadas con herramientas tecnológicas divertidas, es por ello la gran importancia que tiene el tema a investigar que ayuda en gran parte a un óptimo aprendizaje significativo en estudiantes de sectores rurales, que son la parte fundamental para empezar a ver resultados positivos en esta nueva modalidad de educación digital.

Es justamente por lo expuesto que las estrategias metodológicas desde un enfoque innovador tecnológico, son fundamentales al momento de plantear las actividades del currículo educativo pues cada una de ellas contribuye en la planificación docente y el ejercicio de sus acciones en su tarea de enseñanza, se debe motivarlos para que asocien los contenidos con actividades que impliquen juego y hacerlo consciente de lo que hace para que lo asuma con responsabilidad, pero sobre todo con eficacia. 


\section{Enfoque lúdico}

En relación al enfoque lúdico hace referencia a una serie de juegos didácticos que brinda la oportunidad de desarrollar de mejor manera determinadas habilidades o destrezas que poseen las personas desde tempranas edades, mediante este enfoque las personas suelen aprender más rápido y hasta alcanzar su aprendizaje significativo logrando a su vez un desarrollo humano excelente, es así que para (Martinez, 2008), la lúdica podría ser reconocida como una dimensión que posee el ser humano, así como un factor que ayude a decidir su mejor desarrollo, tomando en cuenta que a mayor posibilidad de expresión lúdica un individuo corresponderá mejores posibilidades de aprendizaje.

De tal manera este enfoque brinda un valor específico en la enseñanza que mantiene por el simple hecho de que se podría combinar la colaboración con la colectividad, la distracción y creatividad en el aula, para poder obtener resultados suficientes en situaciones problemáticas en el contexto educativo, por lo tanto los entornos virtuales de aprendizaje son referidos a escenarios educativos cuya base es la tecnología sirviendo como mediadora que favorece en el aprendizaje, a su vez representan una posibilidad en lo que se puede enunciar hasta relacionar con diversas áreas del conocimiento, así mismo tiene como finalidad el desarrollo de las propuestas desde una dimensión científica o socioafectiva.

\section{Entornos Virtuales de Aprendizaje (EVA)}

En cuanto a las experiencias de enseñanza- aprendizaje que puede llevar a cabo mediante EVA sería un crecimiento circunstancial de tiempo y espacio que son muy diferentes al entorno educativo clásico; posteriormente podría dar lugar a algunas categorías de modos de aprendizaje y enseñanza debido a que atiende la concurrencia y la sincronía de ciertos espacios sean de parte del profesor o del alumno. Se procederá a tomar en cuenta nuevos escenarios educativos por lo que es necesario acaparar los 
procesos de enseñanza-aprendizaje autónomo y que sean mediante la comunicación y actividades colaborativas poniendo énfasis en fundamentos online.

Para (Galvao \& Nikolovska, 2014), destaca los aspectos más importantes que se pueden combinar en los entornos virtuales entre los principales están los recursos utilizados, procesos a seguir y la práctica diaria, todos estos tres ayudan a obtener mejores resultados en el aprendizaje del alumno, son tan importantes que contribuye a una mejor calidad de educación, para saber los avances y mejoras en el estudiante es necesario realizar una evaluación utilizando herramientas digitales, es por ello la importancia de EVA para un excelente rendimiento escolar.

\section{Estrategias Metodológicas}

Según (Choquichanca-Lavado \& Inga-Arias, 2020), considera que las estrategias metodológicas son el proceso que ayudan en la selección, adaptación y aplicación de habilidades, conocimientos y metodologías, las cuales tienen el propósito de llegar a un aprendizaje significativo en los alumnos. Ahora bien, relacionándolo con el juego en los entornos digitales y con enfoques de aprendizaje se convierten en herramientas de enseñanza capaz de motivar al educando por su capacidad de ser inmersivo, incluso se ha considerado que este tipo de actividades permiten al estudiante tener experiencias distintas y de gran desafío que les dejan grandes satisfacciones, por lo que se logra compromiso y motivación durante el aprendizaje.

\section{Herramientas Digitales}

Por otra parte las herramientas digitales son muy útiles en la tarea pedagógica en el proceso de enseñanza-aprendizaje que facilitan tanto al docente como al alumno permitiendo reconfigurar las prácticas educativas, pues su dinámica plantea destemporizar y desterritorializar la relación socioeducativa, de manera que se pueden plantear nuevas formas para desarrollar el pensamiento, otras alternativas de actuación 
y relacionamiento en un entorno conocido como sociedad red, misma que requiere de acciones formativas constantes, potenciamiento de la inteligencia colectiva y un conocimiento democratizado a favor de más personas.

Para (Bastidas-Guerreo, 2013), esta propuesta pedagógica de las herramientas digitales se planteó con la intención de atender a las necesidades que presenta la educación virtual, por lo que ha integrado postulados del aprendizaje virtual, pedagogía de la información y también de la conexión para contribuir a un proceso formativo cada vez más popularizado. Ante lo expuesto, es posible considerar que las teorías del aprendizaje basadas en el constructivismo, enactivismo, conectivismo y la del cyber espacio son fundamentales en el contexto actual, donde la educación sucede en un entorno netamente digital debido a la pandemia por COVID-19 que desde hace dos años modificó las prácticas educativas.

Por lo tanto, cada postulado permite al educador basar sus acciones pedagógicas en fundamentos claros y de gran valor en el desarrollo del individuo de manera que se puede reescribir el modelo de educación en la actualidad por medio de la incorporación de las TICs, potenciando la experiencia del educando en los entornos virtuales para un aprendizaje significativo que le permita una verdadera interiorización de la información y desarrollar nuevos conocimientos.

Asimismo, (Johnson et al. 2006), mencionan que para lograr un proceso de enseñanza aprendizaje se debe implementar el uso de herramientas tecnológicas de la nueva revolución digital, mismos que deben ayudar en la participación, desarrollo del pensamiento, interacción y una buena reflexión en los estudiantes, para poder cambiar una educación tradicional a una educación moderna, es un reto con interrogantes y dificultades, tanto para profesores y alumnos ante la situación de tecnologías evolutivas cambiantes.

Es importante tener presente que los niños y niñas tienen obligaciones y derechos que deben ser respetados al momento de utilizar una estrategia metodológica. De acuerdo al 
Reglamento de la Ley Orgánica de Educación Intercultural (Del-pozo-Barrezueta, 2011), menciona que todos los estudiantes tienen la obligación de buscar la excelencia en la educación y que pueden demostrar honestidad e integridad académica en el desempeño de sus funciones.

Asimismo, están obligados a demostrar adecuadamente sus puntos de vista siempre respetando las opiniones de los demás. Por su parte el (Código de la Niñez y Adolescencia, 2013), señala que se debe lograr un desarrollo de la personalidad, de las actitudes y especialmente de la capacidad intelectual y físico del estudiante hasta alcanzar su más alto potencial dentro de un entorno recreativo y afectuoso.

\section{METODOLOGÍA}

El presente trabajo investigativo fue de tipo descriptivo, exploratorio, de campo, bibliográfico, no experimental, de cohorte transversal, con un enfoque mixto, es decir cualitativo y cuantitativo que buscó recoger, analizar e interpretar los datos obtenidos en el trabajo de investigación. Como lo indica (Zafra-Galvis, 2006), que todos pueden idear su propio método dependiendo del tipo de información que deseen realizar y en base al desarrollo del conocimiento sobre el tema a examinar y los objetivos que se persiguen, mediante el uso de diferentes metodologías se puede conseguir obtener información real, continuar con la indagación del tema, investigar el centro del problema y dar soluciones a las dificultades presentadas.

Por otra parte, la investigación se realizó a los docentes de las Escuelas Pluridocentes de la Parroquia de Checa de la Ciudad de Cuenca, del Circuito 18-19, la cantidad total de docentes encuestados fueron 13, de los cuales 12 son mujeres y 1 hombre, debido a la existencia de pocos docentes la aplicación de la investigación se realizó a toda la población y no se procedió con ningún tipo de muestra. Según (Ventura-Leon (2017) señala que el universo a investigar puede ser de manera general muy grande e 
inaccesible y otra que tiene una cantidad pequeña de elementos y se puede realizar con facilidad el desarrollo de la investigación.

La técnica utilizada para la recolección de datos fue encuesta a docentes, y como instrumento se aplicó el uso de un cuestionario con 13 preguntas con opciones de Escala de Likert, luego los resultados de las encuestas fueron subidas a un software Excel SPSS para Windows, mismos que fueron validados por el Método Alfa de Cronbach con un valor de 0,819, finalmente para el análisis y recolección de datos se realizó mediante tablas dicotómicas (frecuencia y porcentaje), para establecer las relaciones existentes entre las variables del trabajo de investigación se utilizó la prueba de Chi cuadrado.

\section{RESULTADOS}

Posteriormente, se procedió a un análisis de las preguntas de la encuesta realizada a los docentes en el programa SPSS de forma individual y cruzando variables independientes y dependientes, tambien se pudo evidenciar que las variables en el test de Shapiro Wilk fueron paramétricas es decir menos de 0,05 logrando los siguientes resultados:

\section{Tabla 1.}

Herramientas virtuales en la actualidad.

\begin{tabular}{llcc}
\hline & & Frecuencia & Porcentaje válido \\
\hline Válido & De acuerdo & 4 & 30,8 \\
& Totalmente de acuerdo & 9 & 69,2 \\
\cline { 2 - 4 } & Total & 13 & 100,0 \\
\hline
\end{tabular}

Fuente: Encuesta.

En la actualidad se ha podido dar a conocer que el mayor porcentaje representado con un $69,2 \%$ de la población encuestada se encuentra totalmente de acuerdo en el uso de las diversas herramientas virtuales que en la actualidad existen; de manera contraria únicamente un 30,8\% del grupo focal ha considerado pertinente el uso de dichas 
herramientas en el ámbito educativo, gracias a una variedad de consecuencias que eso pudiese acarrear.

En cuanto al aspecto utilizado para poder determinar la posible relación existente entre las variables y las preguntas propuestas en la encuesta, se ha implementado un análisis correlacional, siendo así que dicho análisis estadístico se empleó el programa estadístico SPSS.

Las hipótesis que han sido planteadas son las siguientes:

Ho. No hay relación entre las herramientas virtuales y el uso de metodologías activas.

H1: Si hay relación entre las herramientas virtuales y el uso de metodologías activas.

Tabla 2.

Relación de las herramientas virtuales y el uso de metodologías activas.

\begin{tabular}{|c|c|c|c|c|c|}
\hline & & \multicolumn{3}{|c|}{ Frecuencia del uso de metodologías activas } & \multirow[b]{2}{*}{ Total } \\
\hline & & Siempre & $\begin{array}{c}\text { Casi } \\
\text { siempre }\end{array}$ & A veces & \\
\hline \multirow[t]{2}{*}{$\begin{array}{l}\text { Herramientas virtuales } \\
\text { en la actualidad }\end{array}$} & $\begin{array}{l}\text { Totalmente de } \\
\text { acuerdo }\end{array}$ & 1 & 8 & 0 & 9 \\
\hline & De acuerdo & 1 & 2 & 1 & 4 \\
\hline \multicolumn{2}{|c|}{ Total } & 2 & 10 & 1 & 13 \\
\hline \multicolumn{6}{|c|}{ Pruebas de chi-cuadrado } \\
\hline & & Valor & df & $\begin{array}{c}\text { Significación } \\
\text { asintótica } \\
\text { (bilateral) } \\
\end{array}$ & \\
\hline \multicolumn{2}{|l|}{ Chi-cuadrado de Pearson } & $3,142^{\mathrm{a}}$ & 2 & 0,208 & \\
\hline \multicolumn{2}{|c|}{ Razón de verosimilitud } & 3,268 & 2 & 0,195 & \\
\hline \multicolumn{2}{|c|}{ Asociación lineal por lineal } & ,140 & 1 & 0,708 & \\
\hline \multicolumn{2}{|l|}{$\mathrm{N}$ de casos válidos } & 13 & & & \\
\hline
\end{tabular}

Fuente: Encuesta. 
Posteriormente, en nuestra Tabla 2 se obtuvo el cálculo que se da entre las variables mediante la utilización de Pruebas chi-cuadrado se pudo verificar que la significación asintótica seria 0,208 considerada mayor a 0,05, de tal manera que no se rechaza Ho.

\section{Tabla 3.}

Herramientas virtuales en la actualidad *Actividades lúdicas en el área de Lengua y Literatura.

\begin{tabular}{|c|c|c|c|c|c|}
\hline & & & Actividades li & dicas en el área de & \\
\hline & & & Lengue & y Literatura & \\
\hline & & & Siempre & Casi siempre & Total \\
\hline Herramientas virtuales en la & Totalmen & acuerdo & 6 & 3 & 9 \\
\hline actualidad & $\mathrm{De}$ & & 4 & 0 & 4 \\
\hline Total & & & 10 & 3 & 13 \\
\hline Pruebas de chi-cuadrado & & & & & \\
\hline & Valor & df & $\begin{array}{c}\text { Significación } \\
\text { asintótica } \\
\text { (bilateral) }\end{array}$ & $\begin{array}{c}\text { Significación } \\
\text { exacta } \\
\text { (bilateral) }\end{array}$ & $\begin{array}{c}\text { Significación } \\
\text { exacta } \\
\text { (unilateral) }\end{array}$ \\
\hline Chi-cuadrado de Pearson & $1,733^{\mathrm{a}}$ & 1 & 0,188 & & \\
\hline Corrección de continuidad & ,364 & 1 & 0,546 & & \\
\hline Razón de verosimilitud & 2,588 & 1 & 0,108 & & \\
\hline Prueba exacta de Fisher & & & & 0,497 & 0,294 \\
\hline Asociación lineal por lineal & 1,600 & 1 & 0,206 & & \\
\hline
\end{tabular}

Fuente: Encuesta.

No hay relación entre las herramientas virtuales y el uso de metodologías activas y a su vez se rechaza la $\mathrm{Ha}$. Si hay relación entre las herramientas virtuales y el uso de metodologías activas, es decir, se afirma que la hipótesis referida es nula.

De la misma forma se han planteado hipótesis ante esta posible relación:

Ho. Si existe relación entre las herramientas virtuales y las actividades físicas.

H1. No existe relación entre las herramientas virtuales y las actividades físicas. 
En la siguiente Tabla de datos estadísticos se ha dado a conocer que la significación asintótica de Chi cuadrado es 0,188 manifestado su valor superior a 0,05 ; trayendo a colación el no rechazo Ho. Si existe relación entre las herramientas virtuales y las actividades físicas; así como el rechazo absoluto a la Ha. No existe relación entre las herramientas virtuales y las actividades físicas; dándonos como resultado una vez más la afirmación en hipótesis nula.

Por consiguiente, se propuso para los datos estadísticos siguientes las siguientes hipótesis:

Ho. Si existe relación entre las herramientas virtuales y Kahoot.

H1. No existe relación entre las herramientas virtuales y Kahoot.

\section{Tabla 4.}

Herramientas virtuales en la actualidad *Kahoot para la enseñanza de Lengua y Literatura.

\begin{tabular}{llcccc}
\hline & & \multicolumn{2}{c}{ Kahoot para la enseñanza de Lengua y Literatura } & \\
\cline { 2 - 4 } & $\begin{array}{c}\text { Totalmente de } \\
\text { acuerdo }\end{array}$ & De acuerdo & $\begin{array}{c}\text { Medianamente en } \\
\text { desacuerdo }\end{array}$ & Total \\
\hline $\begin{array}{l}\text { Herramientas virtuales } \\
\text { en la actualidad }\end{array}$ & $\begin{array}{l}\text { Totalmente de } \\
\text { acuerdo } \\
\text { De acuerdo }\end{array}$ & 3 & 6 & 0 & 9 \\
Total & 1 & 2 & 1 & 4 \\
\hline
\end{tabular}

Pruebas de chi-cuadrado

\begin{tabular}{lccc} 
& Valor & df & $\begin{array}{c}\text { Significación asintótica } \\
\text { (bilateral) }\end{array}$ \\
\hline Chi-cuadrado de Pearson & $2,438^{\mathrm{a}}$ & 2 & 0,296 \\
Razón de verosimilitud & 2,552 & 2 & 0,279 \\
Asociación lineal por lineal & 0,857 & 1 & 0,355 \\
\hline $\mathrm{N}$ de casos válidos & 13 & & \\
\hline
\end{tabular}

Fuente: Los autores. 
A continuación, para la tabla 4 se alcanzó un 0,296 en lo que respecta a la significación asintótica, correspondiendo a un valor mayor a 0,05 de lo permitido; ocasionando de tal manera una vez más el rechazo de Ho. Si existe relación entre las herramientas virtuales y Kahoot; seguido del total rechazo de la Ha. No existe relación entre las herramientas virtuales y Kahoot; revelando así una hipótesis nula.

Para culminar con todas las variables y su relación, se ha establecido las siguientes hipótesis:

Ho. Si existe relación entre las herramientas virtuales y Quizziz.

H1. No existe relación entre las herramientas virtuales y Quizziz.

\section{Tabla 5.}

Herramientas virtuales en la actualidad *Quizizz para la enseñanza de Lengua y Literatura.

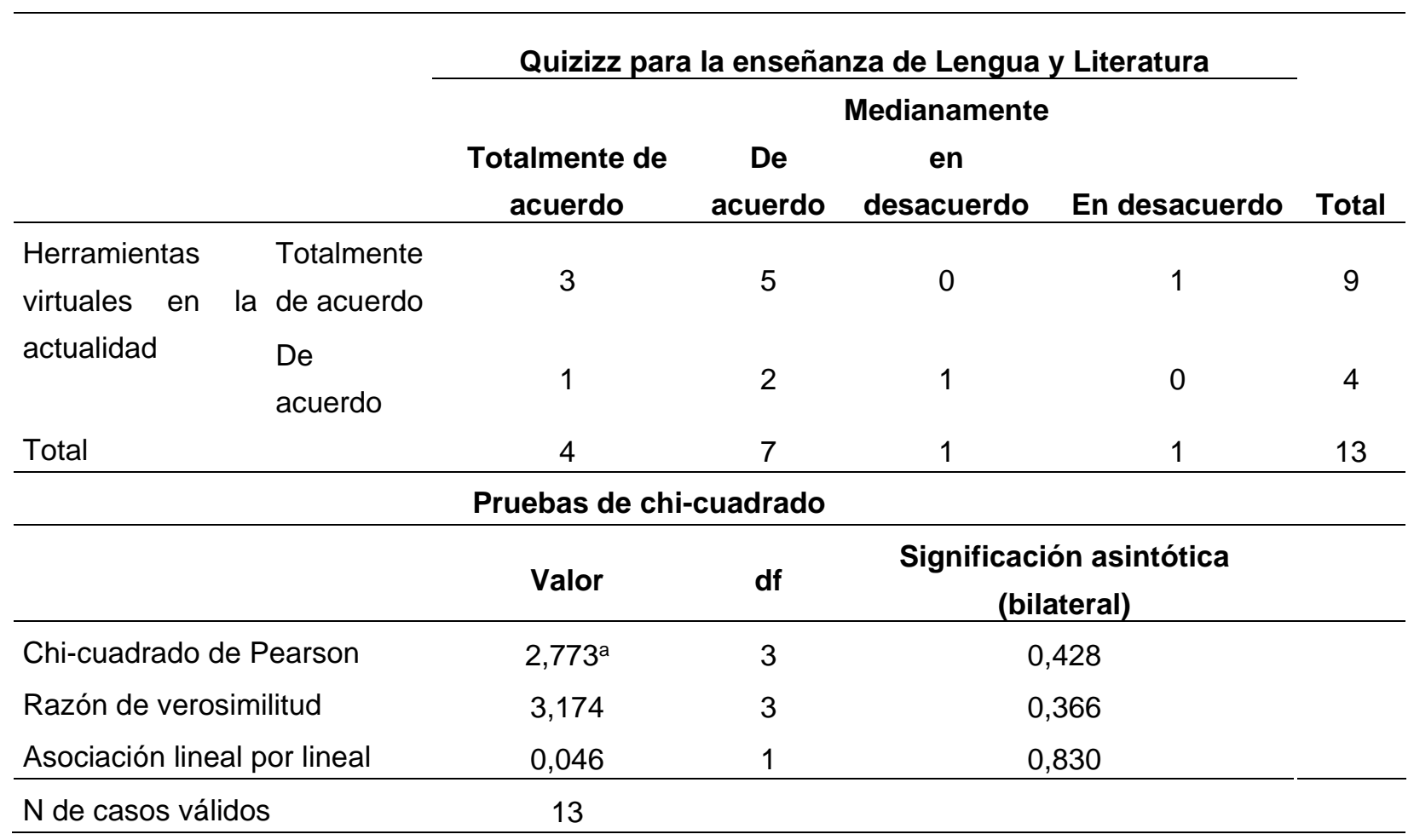

Fuente: Los autores. 
Ahora bien, en la última tabla lograda con datos estadísticos se pudo evidenciar un 0,428 en la significación asintótica lo que concierne un porcentaje mayor a 0,05 de lo correspondido; atribuyendo el respectivo no rechazo a Ho. Si existe relación entre las herramientas virtuales y Quizziz, así como el correcto rechazo a la Ha. No existe relación entre las herramientas virtuales y Quizziz; otorgando la afirmación de una hipótesis nula. En conclusión, tras obtener cada uno de los valores estadísticos en este estudio con respecto al análisis de la posible relación existente entre las variables se puede mencionar que todos los valores albergan una mínima diferencia considerando que dichos valores de la significación asintótica (bilateral) de Chi-cuadrado de Pearson son mayores a 0,05; de tal manera que a nivel general se puede afirmar en el estudio que la hipótesis es nula, por ende no existe ningún tipo de relación entre las variables que fueron analizadas.

Es así que como referencia al análisis estadístico aplicado es necesario conocer que (Quevedo-Ricardi, 2011), en una de sus publicaciones ha manifestado que esta prueba también es conocida como ji-cuadrado dado a que su nivel de distribución de probabilidad hace referencia al mismo nombre, además manifiesta que este tipo de pruebas ayuda a realizar pruebas de hipótesis con relación a las distribuciones de frecuencia, de tal manera que dicha prueba da un contraste a las frecuencias que hayan sido observadas con las frecuencias esperadas de acuerdo con la hipótesis nula.

Por otro lado, (Hernández-de-la-Rosa et al. 2017), da a conocer que este tipo de pruebas estadísticas son muy utilizadas cuando se va a realizar un análisis entre dos o más grupos o a su vez entre dos o más variables, a partir de ello fue convertida en una prueba aceptable y aplicable a diversos usos, siempre y cuando se trata de disponer datos independientes de tipo nominal; dicha prueba brinda un test general con respecto a la existencia de algunas diferencias presentadas en las categorías de los datos de la variable independiente. 


\section{PROPUESTA}

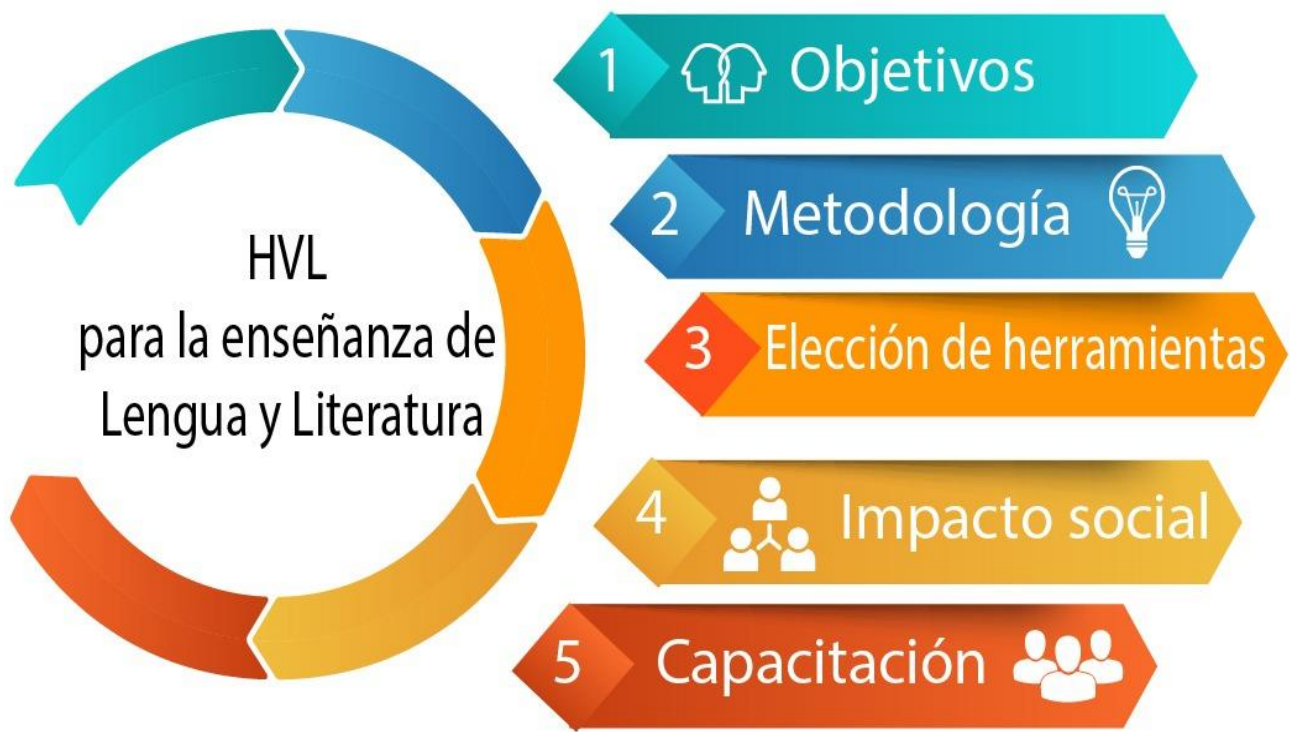

Figura 1. Herramientas virtuales lúdicas para la enseñanza de Lengua y Literatura (HVL). Elaboración: Los autores.

Partiremos manifestando la función destacable que en la actualidad cumplen cada una de las herramientas digitales lúdicas, en el proceso de enseñanza y aprendizaje dentro de cada institución educativa no solo en nuestro país sino a nivel mundial; gracias a su gran capacidad de flexibilidad de manera variada, es decir pueden ser tomadas como un objeto de aprendizaje, al igual que un medio de aprendizaje y totalmente como un apoyo para el aprendizaje.

Por lo tanto, desarrollar una propuesta adecuada donde involucre esta temática respondiendo cada una de las necesidades e interrogantes existentes sería fundamental; es por ello que se ha considerado primordial establecer como objetivo esencial el promover la enseñanza dinámica del área de Lengua y Literatura a través de la implementación de herramientas digitales lúdicas para garantizar un aprendizaje significativo y funcional. 
Es por ello que las herramientas digitales lúdicas escogidas en la siguiente propuesta se han caracterizado por brindar varios beneficios entre estos tenemos el incentivo del desarrollo constante de todas sus habilidades digitales, además de promover una competitividad dado que al momento de añadir un juego la jornada de clase se convierte en un periodo agradable y por ende fomenta el trabajo colaborativo; en tal sentido que cada una de dichas características aportan al cumplimiento de nuestro único objetivo planteado.

Como herramientas seleccionadas en esta propuesta tenemos Kahoot y Quizizz, señaladas como unas de las principales herramientas digitales lúdicas en la actualidad y especialmente adecuada para estudiantes desde los 5 años hasta los 12 años tomando en cuenta para la implementación debido a que es su funcionamiento y utilización es fácil y práctica, garantiza en gran parte una mayor participación de los alumnos ya que sienten una motivación superior hacia nuevos conocimientos o reforzar todos aquellos que ya los poseen; siempre y cuando también sepan controlar su tiempo para realizar cada una de las actividades.

Mediante la aplicación de la presente propuesta de estudio se pretende plasmar cada uno de los beneficios no solo en el ámbito educativo, sino llegar a tener un alcance mayor a nivel social; poniendo énfasis en el acceso a internet que no lo poseen muchos niños/as o adolescentes de tal forma que en nuestra sociedad debemos tratar de sobrellevar uno de los retos más importantes con respecto a dicho estudio, haciendo énfasis en la creación de algunas políticas sociales que brinden la oportunidad de aprovechar al máximo cada una de las herramientas digitales lúdicas no solo dentro de la institución educativa sino en cada uno de los hogares manteniendo de esta manera el propósito de adaptarse a nuevos cambios en la sociedad actual.

Aunque las herramientas digitales seleccionadas son tomadas como las mejores, no son posibles utilizarlas de manera Offline, en este sentido se comprende necesario tomar como un complemento adecuado la implementación de algunas aplicaciones en relación 
a la siguiente propuesta desarrollada; en tal sentido se partirá mencionado a Khan Academy una de las aplicaciones digitales destacables para el aprendizaje de varias asignaturas educativas, su uso ha sido totalmente recomendado desde los 5 años en adelante, no tiene ningún costo y está disponible para todo tipo de dispositivo móvil; como ya se mencionó se caracteriza principalmente para el aprendizaje de varias materias en niños desde la Educación Inicial hasta los alumnos de Bachillerato, gracias a que permite a los mismos seleccionar todos los videos educativos favoritos y descargarlos para observarlos en cualquier momento sin mantener una conexión a internet, en el caso de los más pequeños existen recursos offline para reforzar su aprendizaje como el alfabeto, números, cuentos, entre otros.

Para ello la capacitación práctica mediante un tutor se hace primordial, mediante el cual se busca crear un entorno virtual lúdico que genere interacción entre el docente y el alumno empezando desde un conocimiento básico en el manejo de las herramientas, fomentando el autoaprendizaje e implementando investigaciones permanentes para lograr una motivación creativa y participativa.

\section{CONCLUSIONES}

Una vez finalizada la investigación se ha logrado concluir que la utilización de herramientas digitales lúdicas en el ámbito educativo y especialmente en la enseñanza por parte de los educadores, cada vez va siendo más evidente ya que puede ser la forma más segura al momento de involucrar a los alumnos en una experiencia educativa dinámica y atrayente. A nivel mundial los docentes acaban de empezar a profundizar el conocimiento con respecto a ello, siendo muy aprovechadas todas estas herramientas disponibles de manera que aporten a un trabajo mucho más entretenido y de mayor provecho.

La educación digital implica una serie de estrategias metodológicas de enseñanza y aprendizaje por lo que los docentes deben conocerlas, pero sobre todo saber cómo 
ponerlas en práctica en este contexto tan exigente, cambiante y estresante para la sociedad por el COVID-19. Y es que para los estudiantes tampoco ha sido fácil adoptar una nueva modalidad de estudio, incluso a pesar de ser más de un año desde su primera clase digital, pues esto ha implicado limitaciones importantes como el contacto físico con sus pares y por ende el desarrollo cognitivo, emocional y creativo.

Por ello se puede decir que los profesores usan herramientas virtuales lúdicas en un nivel bajo, por el hecho que recientemente están conociendo e integrando estos recursos en su enseñanza para ir corrigiendo las falencias presentadas en la educación, se puede incrementar el uso de las mismas mediante capacitaciones constantes y prácticas a los docentes, que constituye una parte primordial para evaluar la efectividad de la herramienta utilizada.

Sin embargo, cabe decir que los docentes requieren de especial capacitación no solo para adquirir el dominio necesario de las TIC si no para ajustar dichos fundamentos metodológicos a la educación digital y esta tarea no es fácil si se toman en cuenta las limitaciones de tiempo, acceso a la tecnología o conexión de internet y también de disposición e interés por hacerlo.

Finalmente se pudo establecer que a nivel nacional y local los docentes optan por emplear herramientas digitales lúdicas como Kahoot y Quizizz dado que se tratan de herramientas fáciles de acceder y su implementación en la jornada de clase seria rápida, así como el uso de todos sus recursos educativos son bastante prácticos. De este modo que al educador le resulte mucho más sencillo sobrellevar un seguimiento de sus estudiantes contrastando a su vez los avances alcanzados; por lo que lograrían elevar la motivación e interacción entre los mismos hasta presenciar una participación superior en la jornada educativa diaria. 


\section{FINANCIAMIENTO}

No monetario.

\section{AGRADECIMIENTOS}

A la Universidad Católica de Cuenca; por impulsar el desarrollo de este proceso investigativo desde la praxis educativa.

\section{REFERENCIAS CONSULTADAS}

Adams, S., Cummins, M., Davis, A., Freeman, A., Hall, C., \& Ananthanarayanan, V. (2017). NMC Horizon Report 2017 Highter Education Edition. [Informe Horizon NMC Edicion de Educacion Superior]. The New Media Consortium, 1(Informe), 36-48. https://n9.cl/em457

Albornoz, E., \& Guzman, M. (2016). Mediante estimulación desarrollo cognitivo mediante estimulación en niños de 3 años. Centro de Desarrollo Infantil nuevos horizontes [By stimulating cognitive development through stimulation in 3-year-old children. New Horizons Child Development Center]. Revista Cientifica Multidisciplinar de La Universidad de Cienfuegos, 4(Articulo), 186-192. https://n9.cl/r7d5j

Bastidas-Guerreo, O. (2013). Educativa, Enseñanza-Aprendizaje y Evaluacion en el Ciberespacio: Una nueva realidad. [Educational, Teaching-Learning and Assessment in Cyberspace: A new reality]. https://n9.cl/u256t

Cabrera-Baculima, J. (2016). Importancia de las Tic para el mejoramiento del proceso enseñanza aprendizaje en educacion general basica. [Importance of ICT for the improvement of the teaching-learning process in basic general education] [Universidad de Cuenca]. http://dspace.ucuenca.edu.ec/handle/123456789/24138

Camargo-Miranda, G., Cortez-Baqueth, L., y Ramirez-Torres, D. (2017). La Lúdica como estrategia Pedagógica para mejorar los procesos de motivación de adolescentes del grado $9^{\circ} \mathrm{en}$ la Institución Educativa san Felipe Neri de la Ciudad de Cartagena. [Play as a Pedagogical strategy to improve the motivation processes of 9th grad [Fundacion Universitaria los Libertadores]. https://n9.cl/kbzj8 
CEPAL-UNESCO. (2020). La Educación en tiempos de Pandemia de COVID- 19. [Education in times of the COVID-19 Pandemic]. Recuperado de https://n9.cl/b613e

Choquichanca-Lavado, R, \& Inga-Arias, M. (2020). Estrategias metodológicas docentes en comprensión lectora y rendimiento académico en el área de Comunicación [Methodological teaching strategies in reading comprehension and academic performance in the area of communication]. Desde el Sur, 12(2), 479500. https://dx.doi.org/10.21142/des-1202-2020-0027

CNA. (2013). Los Niños, Niñas y Adolescentes como sujetos de Derechos. [Children and Adolescents as subjects of Rights]. Codigo de La Niñez y La Adolescencia. https://n9.cl/fokqg

Código de la Niñez y Adolescencia. (2013). Recuperado de https://n9.cl/fokqg

Del-pozo-Barrezueta, H. (2011). Ley Organica de Educacion Intercultural. [Orgnic Law of Education Intercultual]. Registro Oficial Órgano Del Gobierno Del Ecuador. https://n9.cl/at9p

Galvao, M., \& Nikolovska, M. (2014). Quality assurance in vocational education and training [Garantía de calidad en la educación y formación profesionales]. https://n9.cl/hy1ar

Hernández-de-la-Rosa, Y., Hernández Moreno, V, Batista Hernández, N, \& Tejeda Castañeda, E. (2017). ¿Chi cuadrado o Ji cuadrado? Medicentro Electrónica, 21(4), 294-295.

Johnson, J., Chapman, C., \& Dyer, J. (2006). Pedagogy and Innovation in Education with Digital technologies. [Pedagogía e Innovación en Educación con Tecnologías Digitales]. Formatex, 2(Informe), 2-5. https://n9.cl/m5jln

Martinez, M. (2008). Aprendizaje, Servicio y Responsabilidad Social sobre las Universidades. [Learning, Service and Social Responsibility on Universities]. Centro Español de Derechos Reprográficos, 3(Informe), 11-27. https://n9.cl/0zip

MINEDUC. (2020). Plan Educativo COVID-19 [COVID-19 Educational Plan]. Recuperado de https://educacion.gob.ec/plan-educativo-covid-19/

Piedra-Vera, S. (2018). Factores que aportan las actividades lúdicas en los contextos Educativos. [Factors that contribute to recreational activities in educational contexts]. Revista Cognosis, 3(Informe), 4-10. https://n9.cl/u7az 
Quevedo-Ricardi, F. (2011). La prueba de ji-cuadrado. [The chi-square]. Medwave. https://doi.org/10.5867/medwave.2011.12.5266

Raso-Sanchez, F., Aznar-Diaz, I., y Caceres-Reche, M. (2014). Integración de Tecnologías de la Información y Comunicación: Estudio Evaluativo en la Escuela Rural Andaluza España. [Integration of Information and Communication Technologies: Evaluation Study at the Andalusian Rural School in Spain]. Revista de Medios y Educacion, 45(Articulo), 51-64. https://n9.cl/x5311

Ventura-Leon, J. (2017). ¿Población o Muestra?: Una diferencia necesaria. [Population or Sample ?: A necessary difference]. Revista Cubana de Salud Publica, 43(Articulo), 648-649. https://n9.cl/aji8y

Villafuerte, P. (2020). Educación en Tiempos de Pandemia COVID-19 y equidad en el Aprendizaje. [Education in Times of the COVID-19 Pandemic and Equity in Learning]. Instituto Para El Futuro de La Educacion. https://n9.cl/mvft

Zafra-Galvis, O. (2006). Tipos de Investigación. [Types of research]. Revista Científica General José María Córdova, 4(Informe), 13-14. https://n9.cl/8d6hq 